\title{
Sling de la arteria pulmonar en paciente preescolar con compromiso de la vía aérea
}

\author{
M. Alejandra Monroy-Cárdenas ${ }^{1 *}$, Ricardo Hernández-Sarmiento ${ }^{2}$ y Oscar U. Barón-Puentes ${ }^{3}$

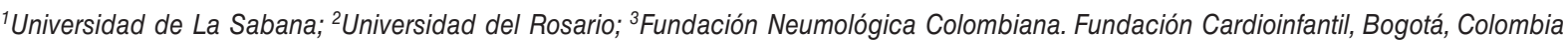

\section{Resumen}

Introducción: El sling de la arteria pulmonar izquierda es una rara malformación congénita del arco aórtico en la que la rama pulmonar izquierda se origina a partir de la arteria pulmonar derecha. Generalmente se sitúa entre la tráquea y el esófago, que frecuentemente la comprimen. Dependiendo del grado de compromiso de la vía aérea, el esófago y las anomalías cardíacas asociadas, las manifestaciones clínicas varían. El tratamiento de esta malformación es quirúrgico; con el paso de los años, este abordaje ha mejorado, y se ha logrado una disminución de la morbilidad y mortalidad en esta condición. Caso clínico: Se presenta el caso de una paciente preescolar con hallazgo de sling de la arteria pulmonar izquierda con compromiso de la vía aérea. Conclusiones: Después de la corrección quirúrgica, se logró una respuesta clínica favorable y una mejoría significativa en la vía aérea, evidenciada en los hallazgos en la fibrobroncoscopia, sin necesidad de intervenciones adicionales.

Palabras clave: Anillo vascular. Cabestrillo (sling). Anomalía congénita de la arteria pulmonar. Disfagia. Estenosis de la tráquea.

\section{Pulmonary artery sling in preschool patient with airway compromise}

\section{Abstract}

Background: Left pulmonary artery sling is a rare congenital malformation of the aortic arch in which this artery originates from the right pulmonary artery and courses over the trachea and the esophagus. Clinical manifestations vary depending on the degree of airway compromise, the esophagus and associated cardiac anomalies. The treatment for this malformation is surgical; over the years, this approach has improved, achieving a decrease in morbidity and mortality in this condition. Case report: A preschool patient with incidental finding of sling of the left pulmonary artery with compromise of the airway is presented. Conclusions: After the surgical procedure, a favorable clinical outcome was observed and a significant improvement in the airway was shown by a fibrobronchoscopy, without any additional intervention.

Key words: Vascular ring. Sling. Congenital pulmonary artery anomaly. Dysphagia. Tracheal stenosis.

Disponible en internet: 11-07-2019 Bol Med Hosp Infant Mex.2019;76:241-245 www.bmhim.com
Fecha de aceptación: 11-07-2019 DOI: 10.24875/BMHIM.19000103 


\section{Introducción}

Las malformaciones del arco aórtico y sus ramas constituyen menos del 1\% de las anomalías congénitas cardiovasculares y se manifiestan típicamente en los primeros años de vida ${ }^{1,2}$. El sling (cabestrillo) de la arteria pulmonar es una rara malformación del anillo vascular en la que la arteria pulmonar izquierda se origina de la parte posterior de la rama pulmonar derecha y se dirige hacia la izquierda ${ }^{3,4}$. El vaso aberrante que resulta de esta condición, aunque es frecuentemente hipoplásico, comprime el esófago por la parte anterior y la tráquea por la parte posterior, lo que puede ocasionar una sintomatología respiratoria o gastrointestinal, según la zona afectada ${ }^{5,6}$. Esta anomalía presenta manifestaciones clínicas variadas que incluyen síntomas respiratorios, como tos, estridor o sibilancias, y digestivos, como disfagia, por lo cual puede pasar desapercibida o confundirse con otras condiciones clínicas.

A continuación, se presenta el caso de una paciente con sling de la arteria pulmonar, sintomática, con cardiopatía congénita asociada que se corrigió con tratamiento quirúrgico.

\section{Caso clínico}

Paciente de sexo femenino de 3 años y 6 meses de edad. Al empezar con la alimentación complementaria, hacia los 6 meses de edad, presentó disfagia, particularmente con la ingesta de sólidos, asociada con tos seca ocasional no cianosante ni emetizante. Como antecedentes prenatales, la paciente es producto de un tercer embarazo controlado: en ecografías obstétricas, se documentó una alteración cardíaca no especificada. Como antecedentes patológicos, se diagnosticaron retardo global en el neurodesarollo, ductus arterioso persistente (DAP), comunicación interventricular (CIV) e hipertensión pulmonar moderada en manejo ambulatorio con furosemida $10 \mathrm{mg}$ vía oral cada $12 \mathrm{~h}$, captopril $10 \mathrm{mg}$ vía oral cada $12 \mathrm{~h}$ y oxígeno por cánula nasal 1/4 I/min por patología de base. La paciente presentaba fenotipo peculiar al examen físico, con fontanela anterior amplia de $2 \mathrm{~cm}$, sialorrea continua y adecuada saturación de oxígeno. En la auscultación cardíaca, se detectó soplo protomesosistólico regurgitante tricuspídeo grado II y S2 intensamente reforzado, sin hallazgos en la auscultación a nivel pulmonar.

Se realizó cateterismo cardíaco a los 3 años y 6 meses con la finalidad de determinar resistencias pulmonares

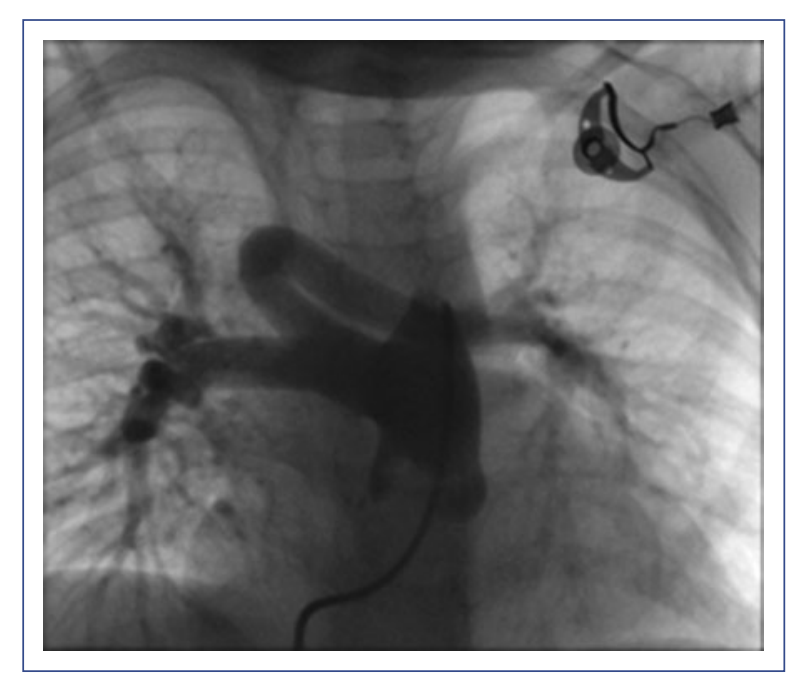

Figura 1. Cateterismo cardíaco prequirúrgico. Sling de la arteria pulmonar izquierda.

y discutir su caso en la junta médico-quirúrgica. Se detectó DAP grande tubular (5 mm), CIV perimembranosa pequeña a moderada $(7 \mathrm{~mm})$, doble vena cava superior con innominada, hipertensión pulmonar hiperquinética $20 \mathrm{mmHg}$ por debajo de la aórtica, con una resistencia pulmonar (Rp) de 6.99 unidades, resistencia sistémica (Rs) de 52.07 unidades (relación Rp/Rs de $0.13)$, circulación pulmonar (Qp) de $6.30 \mathrm{l} / \mathrm{min}$ y circulación sistémica (Qs) de 1.57 l/min (relación Qp/Qs 4.0:1). Además, como hallazgo más significativo, sling de la arteria pulmonar izquierda (Fig. 1).

Se realizó una tomografía axial computarizada de tórax, donde se confirmó el sling de la arteria pulmonar izquierda relacionado con una compresión extrínseca del bronquio fuente izquierdo (Fig. 2). Por estos hallazgos, a la paciente se le realizó una fibrobroncoscopia, lo que evidenció compromiso del calibre de la tráquea, sin estenosis, con colapso dinámico del bronquio fuente izquierdo que comprometía la luz en aproximadamente el $50 \%$ (Fig. 3).

El caso fue discutido en la junta médico-quirúrgica de cardiopediatría, neumopediatría y cirugía cardiovascular, y se decidió realizar la corrección quirúrgica del sling. Por medio de esternotomía media, se realizó la disección de la arteria pulmonar izquierda en todo su recorrido y su reimplante al tronco de la arteria pulmonar. Además, se realizó la sección y ligadura del DAP y auriculotomía derecha con corrección de la CIV por medio de suturas y parche GORE-TEX ${ }^{\circledR}$.

La paciente presentó mejoría de la disfagia y de la tos. Después de 7 meses de la intervención quirúrgica, 


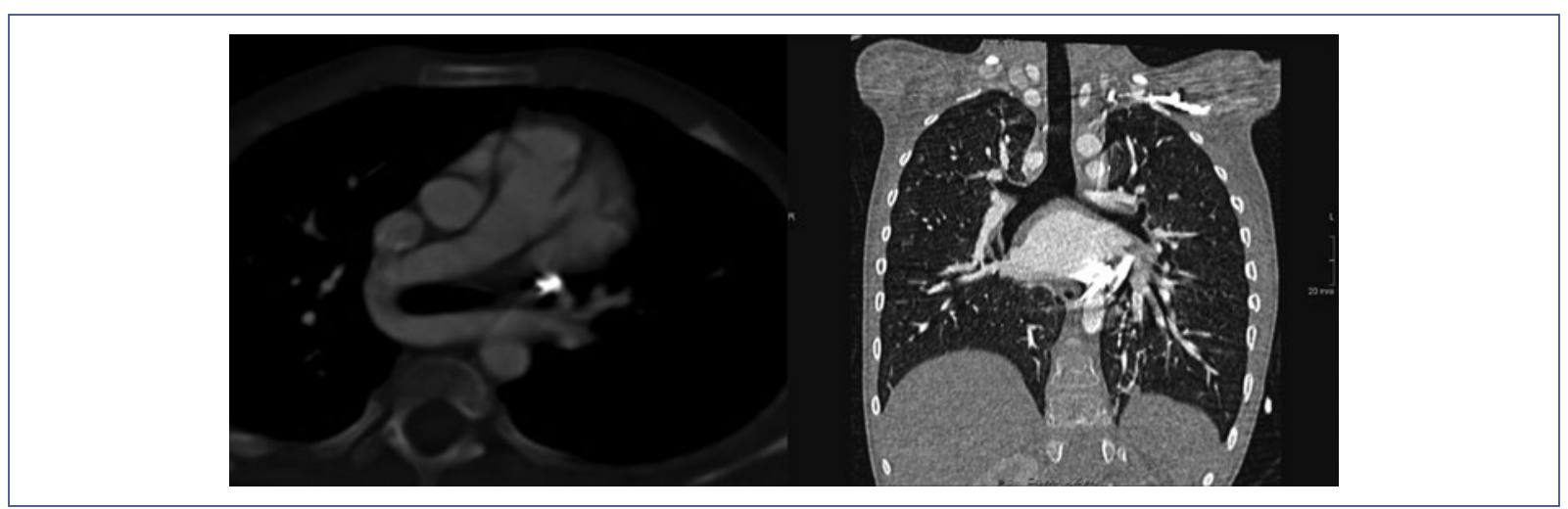

Figura 2. Tomografía axial computarizada de tórax. Evidencia del sling de la arteria pulmonar que compromete la luz de la tráquea.

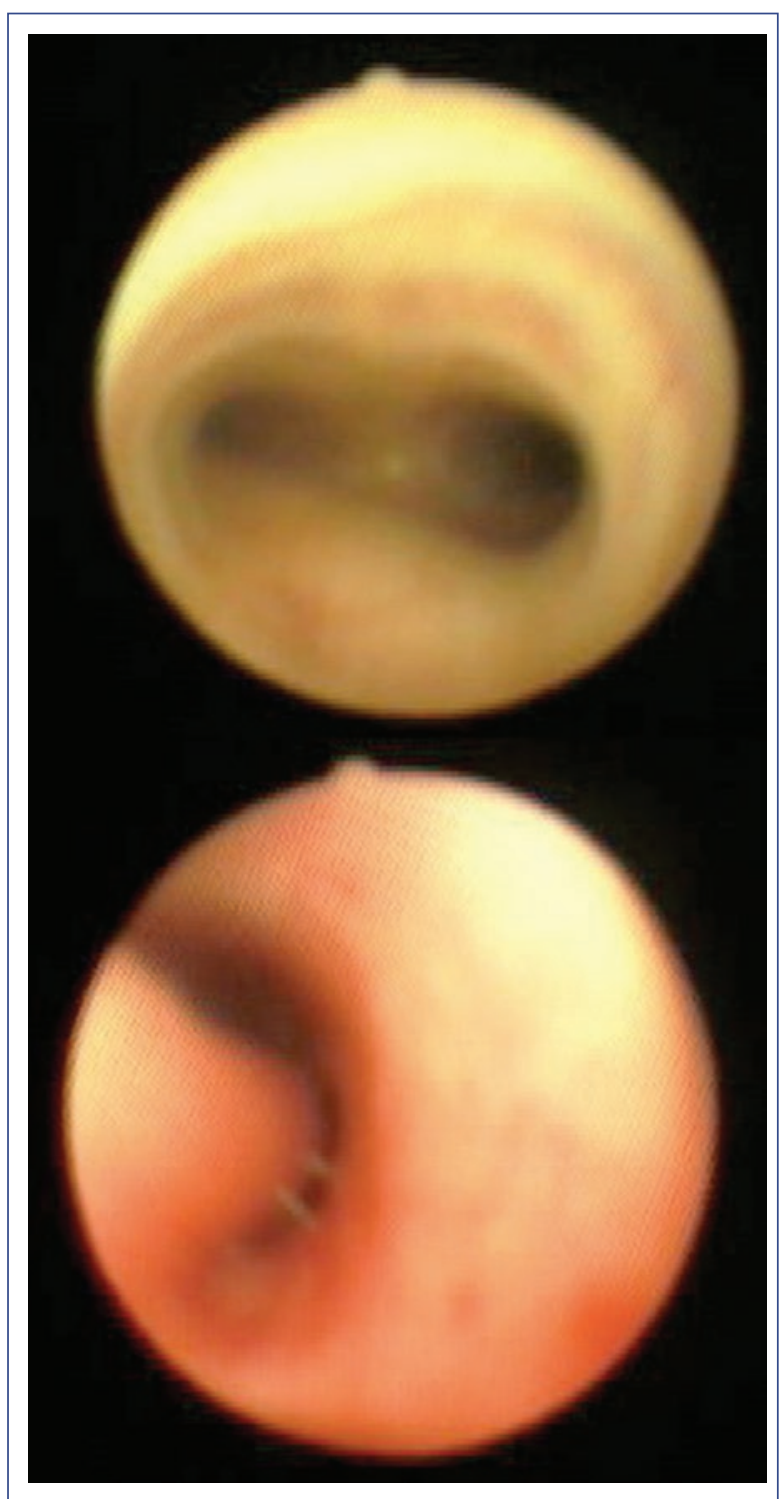

Figura 3. Fibrobroncoscopia prequirúrgica. Compromiso de la luz del bronquio fuente izquierdo. se realizó la fibrobroncoscopia de control, que evidenció una mejoría del colapso de la tráquea y del bronquio fuente izquierdo (Fig. 4), por lo que ya no fue necesario ningún procedimiento quirúrgico adicional.

\section{Discusión}

Las malformaciones del arco aórtico y sus ramas se deben a una alteración precoz en el desarrollo embrionario, y son el resultado de la involución anormal de alguno de los arcos branquiales embrionarios. Dentro de estas anomalías vasculares, se incluye el sling de la arteria pulmonar, que se presenta por la involución del sexto arco aórtico proximal izquierdo y representa menos del $3 \%$ de todas las anormalidades del arco aórtico ${ }^{7-10}$. En un estudio retrospectivo realizado en el Guangdong General Hospital, en China, entre octubre del 2007 y diciembre del 2014, se evaluaron 52,200 pacientes con cardiopatía congénita, de los cuales 47 presentaban sling de la arteria pulmonar izquierda $(0.14 \%)^{11}$.

En el presente caso, la paciente contaba con un diagnóstico antenatal de cardiopatía. Sin embargo, no estaba especificada, por lo que inicialmente no se sospechó la alteración vascular. Comúnmente, los anillos vasculares se encuentran asociados con otros defectos congénitos, por lo que es raro que se presenten de manera aislada: aproximadamente el $50 \%$ de estos casos presenta cardiopatías congénitas, como doble tracto de salida del ventrículo izquierdo, tetralogía de Fallot o defecto septal ventricular y DAP, como en este caso. Del 58 al $83 \%$ de los casos presentan otras anomalías congénitas extracardíacas: en el $50 \%$ de los casos se encuentran anillos traqueales cartilaginosos completos, por lo que pueden presentar traqueomalacia o lobulaciones pulmonares anormales ${ }^{4,8,12}$. 


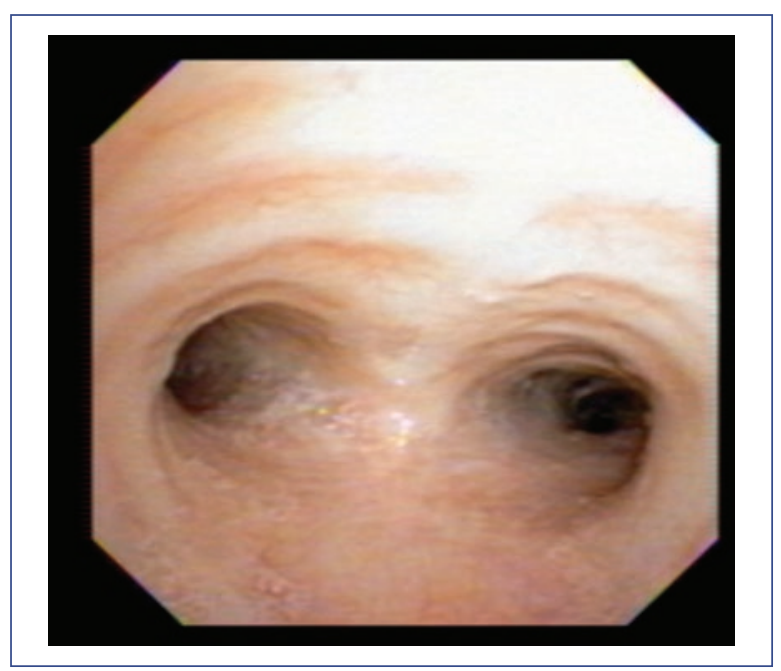

Figura 4. Fibrobroncoscopia posquirúrgica. Normalización de la luz del bronquio fuente izquierdo.

Los anillos vasculares se caracterizan por el curso anómalo de la rama pulmonar izquierda que se origina en la cara posterior de la arteria pulmonar derecha, en lugar del tronco común de la arteria pulmonar, con un trayecto entre el esófago y la tráquea ${ }^{3-5}$.

Las manifestaciones clínicas que provoca este tipo de anillo vascular se basan en el grado de compresión ejercido sobre la vía aérea y el esófago. La paciente del presente reporte mostró disfagia y tos seca ocasional al momento del inicio de la alimentación complementaria, síntomas inespecíficos que no llevaron a la sospecha clínica del anillo vascular. Con el compromiso del calibre a nivel del bronquio fuente izquierdo por el anillo vascular, esta paciente solamente presentaba tos ocasional y nunca requirió internamiento por cuadros respiratorios. La exploración física no fue reveladora, ya que no existía un hallazgo patognomónico que orientara hacia el diagnóstico del anillo vascular. La mayoría de los pacientes pueden presentar soplo cardíaco por la cardiopatía asociada al sling. En este caso, el soplo correspondía a la CIV que padecía la paciente.

En el caso que se presenta, el hallazgo fue incidental, posterior a la realización del cateterismo para determinar resistencias pulmonares. Sin embargo, la presencia del sling de la arteria pulmonar se pudo observar con base en la sospecha clínica. Estudios como la radiografía de tórax o el esofagograma con trago de bario no son específicos de esta patología. La tomografía axial computarizada o la resonancia magnética nuclear de tórax son más útiles para estos casos, ya que muestran tanto la malformación vascular como el compromiso del esófago y de la vía aérea, y se logra determinar la anatomía antes de la intervención quirúrgica (como en el presente caso).

La fibrobroncoscopia se debe realizar para evaluar la vía aérea e identificar el grado de estenosis traqueal o la presencia de anillos traqueales ${ }^{13}$. En esta paciente se identificó el colapso dinámico del árbol bronquial izquierdo por el segmento proximal de la arteria pulmonar izquierda, comprometiendo la luz del mismo. No fue necesaria la dilatación o stent para corregir la obstrucción, ya que la paciente mejoró con la corrección quirúrgica del sling.

Desde la primera corrección, en 1953, el tratamiento de los pacientes sintomáticos es quirúrgico, y ha cambiado de forma muy importante en los últimos años ${ }^{14,15}$. La demostración de un anillo vascular no es indicación quirúrgica por sí misma: es imprescindible tener en cuenta la sintomatología del paciente y hacer una valoración multidisciplinaria. La cirugía de la paciente del caso se realizó para la corrección de las otras cardiopatías y del sling de la arteria pulmonar, con el fin de disminuir la obstrucción generada en el bronquio fuente izquierdo. Este procedimiento se realizó mediante el reimplante de arteria pulmonar izquierda al tronco de la pulmonar; en la misma cirugía, se repararon las otras malformaciones cardíacas asociadas. Después de la cirugía, se observaron resultados satisfactorios, tanto clínicos (mejoría de los síntomas de la paciente) como en la fibrobroncoscopia de control, que presentó mejoría de la obstrucción del calibre del bronquio fuente izquierdo. Las complicaciones posquirúrgicas más comunes reportadas en la literatura son la parálisis del nervio laríngeo recurrente, el quilotórax y el neumotó$\operatorname{rax}^{11,16}$. En este caso, no se presentó ninguna de ellas.

En el estudio retrospectivo realizado por Xie, et al., en 47 pacientes con sling de la arteria pulmonar izquierda se reportó una tasa de mortalidad promedio de $17.9 \%$ después de 5 días de la cirugía. Esta mortalidad es similar a la mencionada en otros reportes, con una tasa que varía del 11 al $45 \%{ }^{11}$. Yong, et al. mostraron un descenso en la mortalidad de los pacientes con este padecimiento cada 10 años, desde 1984 hasta 2011, debido a la mejoría de la técnica quirúrgica. Los autores determinaron mayor sobrevida en los pacientes que no requirieron de cirugía traqueal ${ }^{16}$.

\section{Responsabilidades éticas}

Protección de personas y animales. Los autores declaran que para esta investigación no se han realizado experimentos en seres humanos ni en animales. 
Confidencialidad de los datos. Los autores declaran que han seguido los protocolos de su centro de trabajo sobre la publicación de datos de pacientes.

Derecho a la privacidad y consentimiento informado. Los autores han obtenido el consentimiento informado de los pacientes o individuos referidos en el artículo. Este documento obra en poder del autor de correspondencia.

\section{Conflicto de intereses}

Los autores declaran no tener ningún conflicto de intereses.

\section{Financiamiento}

No se recibió financiamiento para la generación de este artículo.

\section{Agradecimientos}

A las Fundación Neumología Colombiana y a la Fundación Cardioinfantil por facilitarnos las imágenes. A la paciente y su familia por autorizarnos a difundir este caso.

\section{Bibliografía}

1. Domènech $A B$, López J, Rodríguez $P$, Serrano $F$, Fernández B, Gutiérrez $C$, et al. Anillos vasculares, nuestra experiencia en 18 casos. Cir Pediatr. 2014;27:110-6.

2. Tola H, Ozturk E, Yildiz O, Erek E, Haydin S, Turkvatan A, et al. Assessment of children with vascular ring. Pediatr Int. 2017;59:134-40.

3. Gikonyo BM, Jue KL, Edwards JE. Pulmonary vascular sling: report of seven cases and review of the literature. Pediatr Cardiol. 1989;10:81-8.

4. Javia L, Harris MA, Fuller S. Rings, slings, and other tracheal disorders in the neonate. Semin Fetal Neonatal Med. 2016;21:277-84.

5. Berdon WE, Baker DH, Wung J-T, Chrispin A, Kozlowski K, de Silva M, et al. Complete cartilage-ring tracheal stenosis associated with anomalous left pulmonary artery: the ring-sling complex. Radiology. 1984;152:57-64.

6. Sánchez Pérez R, Rey Lois J, Polo López L, Aroca Peinado Á, González Rocafort Á, Pérez González R, et al. Anillos vasculares y compresión traqueo-esofágica: 15 años de experiencia quirúrgica. Cir Cardiovasc. 2016;23:119-24

7. Andrés AS, Carrasco-Moreno Jl. Anillos vasculares y slings [Internet]. Sección de Cardiología Pediátrica. Valencia: Hospital Infantil Universitario La Fe, Valencia. Disponible en: http://www.lisandrobenmaor.com/archivos/33.pdf.

8. Bueno J, Flors L, Mejía M. Anomalías congénitas de las arterias pulmonares: espectro de hallazgos en tomografía computarizada. Radiología. 2017;59:181-270.

9. Lin JH, Chen SJ, Wu MH, Wang JK, Li YW, Lue HC. Right lung agenesis with left pulmonary artery sling. Pediatr Pulmonol. 2000;29:239-41.

10. Collell R, Marimón C, Montero M. Sling parcial de la arteria pulmonar izquierda. Rev Esp Cardiol. 2010;63:850.

11. Xie J, Juan YH, Wang Q, Chen J, Zhuang J, Xie Z, et al. Evaluation of left pulmonary artery sling, associated cardiovascular anomalies, and surgical outcomes using cardiovascular computed tomography angiography. Sci Rep. 2017;7:40042.

12. Backer CL, Mongé MC, Popescu AR, Eltayeb OM, Rastatter JC, Rigsby CK. Vascular rings. Semin Pediatr Surg. 2016;25:165-75.

13. Newman B, Cho Ya. Left pulmonary artery sling-anatomy and imaging. Semin Ultrasound CT MRI. 2010;31:158-70.

14. Collins RT, Weinberg PM, Ewing S, Fogel M. Pulmonary artery sling in an asymptomatic 15-year-old boy. Circulation. 2008;117:2403-6.

15. Backer CL, Russell HM, Kaushal S, Rastatter JC, Rigsby CK, Holinger LD. Pulmonary artery sling: current results with cardiopulmonary bypass. J Thorac Cardiovasc Surg. 2012;143:144-51.

16. Yong MS, d'Udekem Y, Brizard CP, Robertson T, Robertson CF, Weintraub R, et al. Surgical management of pulmonary artery sling in children. J Thorac Cardiovasc Surg. 2013;145:1033-9. 